\title{
Falhas em implantes de quadril
}

\author{
Failures in hip implants \\ Fallos en implantes de cadera
}

\section{Resumo}

A fratura óssea, indubitavelmente, reside em um dos principais problemas associados ao aumento da expectativa de vida da população. Principalmente, as fraturas femorais, as quais demandam por tratamento cirúrgico para redução da fratura por osteossíntese. Nas fraturas femorais com comprometimento grave da estrutura anatômica na epífise proximal, recomenda-se a substituição da articulação por implante total do quadril (THR - Total Hip Replacement). A artroplastia de quadril trata-se de um procedimento largamente utilizado na medicina, contudo, diversas falhas de implantes são reportadas. O objetivo desse trabalho consistiu em apresentar, por meio de uma revisão de literatura, as principais causas que levam os implantes totais de quadril a falharem em uso e elucidar ações de medidas corretivas para evitar tais falhas. Foram identificadas as principais causas de falhas nos implantes: fadiga, afrouxamento, metalose, associados a outros insucessos relacionado ao perfil do paciente. Concluiu-se que os dispositivos de osteossíntese precisam ser elaborados de forma multidisciplinar com parâmetros exigentes, envolvendo ciência médica, projeto de engenharia e seleção de material, com propósito de minorar os riscos de falhas e prevenir acidentes, por conseguinte, evitar ressubmeter o indivíduo a novo procedimento cirúrgico de correção.

Palavras-chave: Osteossíntese; Artroplastia de quadril; Cirurgias; Fraturas femorais; Quadril.

\begin{abstract}
Bone fracture, undoubtedly, is one of the main problems associated with the increase in the population's life expectancy. Mainly, femoral fractures, which require surgical treatment in order to reduce the fracture by osteosynthesis. In case of severe impairment on the proximal epiphysis femoral, complete joint replacement by a total hip implant is recommended. Hip arthroplasty is a procedure widely applied in medicine; however, several implant failures have been reported. This study aimed to present, through a literature review, the main causes that lead total hip implants to failure in use and to elucidate corrective measures to avoid such failures. The leading causes of implant failures identified were: fatigue, loosening, metallosis, in addition to disability and disease related to the patient's profile. In conclusion, a multidisciplinary team shall design osteosynthesis devices. This team needs knowledge and expertise in medical science, engineering design, and material selection. The main purpose is to achieve optimized design parameters to reduce the risk of failure and prevent accidents, therefore avoiding resubmitting the individual to a new surgical correction procedure.
\end{abstract}

Keywords: Osteosynthesis; Hip arthroplasty; Surgeries; Femoral fractures; Hip.

\section{Resumen}

La fractura ósea, sin duda, es uno de los principales problemas asociados al aumento de la esperanza de vida de la población. Principalmente, fracturas de fémur, que requieren tratamiento quirúrgico para reducir la fractura por osteosíntesis. En caso de daño severo en la epífisis femoral proximal, se recomienda el reemplazo articular completo mediante un implante total de cadera. La artroplastia de cadera es un procedimiento ampliamente aplicado en medicina; sin embargo, se han informado varios fallos de implantes. El objetivo de este estudio fue presentar, a través de una revisión de la literatura, las principales causas que llevan a los implantes totales de cadera al fracaso en su uso y dilucidar las medidas correctoras para evitarlos. Las principales causas de falla de los implantes identificadas fueron: fatiga, aflojamiento, metalosis, además de discapacidad y enfermedad relacionada con el perfil del paciente. En conclusión, los dispositivos de osteosíntesis deberán ser diseñados por un equipo multidisciplinario que incluya conocimientos y experiencia en ciencias médicas, diseño de ingeniería y selección de materiales. El objetivo principal es lograr unos parámetros de diseño optimizados con el fin de reducir el riesgo de fallas y prevenir accidentes, evitando así reenviar al individuo a un nuevo procedimiento de corrección quirúrgica.

Palabras clave: Osteosíntesis; Artroplastia de cadera; Cirugías; Fracturas femorales; Cadera. 


\section{Introdução}

Histologicamente, o tecido ósseo é um tecido conjuntivo especializado, cuja matriz apresenta constituintes inorgânico e orgânico. A parte inorgânica é composta por cristais de hidroxiapatita de cálcio, contendo também, além do cálcio, o elemento químico fósforo. Com relação à parte orgânica, essa é constituída predominantemente por colágeno do tipo I e células do tecido ósseo: células osteoprogenitoras, osteoblastos, osteócitos e osteoclastos (Gatner, 2007).

Tais células, fisiologicamente, atuam de forma equilibrada entre atividades de síntese e reabsorção óssea com objetivo de garantir, respectivamente, a manutenção da estrutura óssea e manter os níveis séricos de cálcio adequados no organismo (Gatner, 2007). Portanto, no osso normal, sem presença de alguma manifestação patológica, as células ósseas promovem a homeostase do tecido ósseo, por meio do processo de deposição de cálcio na estrutura esquelética por ação dos osteoblastos, que são responsáveis pela síntese da matriz. Em contrapartida, os osteoclastos desempenham papel na reabsorção óssea, removendo o cálcio depositado na matriz e disponibilizando-o na corrente sanguínea. Trata-se de um constante turnover entre osteoblastos e osteoclastos, os quais funcionam em proporcional velocidade de atuação, a fim de promover a referida homeostase do tecido ósseo (Dourado, 2012).

A fisiologia óssea do organismo, entretanto, sofre alteração desse padrão de funcionamento, mediante ao envelhecimento do indivíduo. Após o pico de massa óssea, que, de acordo com a literatura, é atingido entre 25 e 30 anos de idade, inicia-se, gradativamente, um processo de perda tecidual. Por volta da $3^{\circ}$ década de vida, a relação homeostática perde a proporcionalidade da velocidade de atuação das células ósseas, observa-se, portanto, aumento da função reabsortiva, em detrimento à velocidade de síntese óssea (Dourado, 2012).

Sob essa ótica, é perceptível que, ao decorrer dos anos, a densidade óssea diminuirá progressivamente, assim como, haverá a tendência à rarefação do osso esponjoso. Nesse contexto, a matriz óssea tenderá a ficar mais porosa, apresentando aumento dos espaços intertrabeculares, redução da resistência mecânica e aumento da fragilidade. Consequentemente, a reunião de todos esses resultados culminará no aumento da propensão à fratura osteoporótica (Dourado, 2012).

Dessa forma, a desproporção das relações de velocidade de reabsorção superior à de síntese da matriz óssea promoverá uma repercussão a nível sistêmico, definida como osteoporose. Trata-se de uma doença osteometabólica a qual afeta a estrutura esquelética em virtude de dois eventos: redução da densidade óssea e deterioração da arquitetura do tecido ósseo. Importante ressaltar que, apesar da osteoporose estar intimamente relacionada à senilidade, tende afetar mais a população feminina, pois esse grupo apresenta menor pico de massa óssea e, ainda, encontra-se sujeito às consequências provenientes da menopausa, principalmente, se incidir precocemente (Caputo \& Costa, 2014).

A osteoporose habitua-se a evoluir silenciosamente, portanto, em virtude das manifestações clínicas insidiosas nesse tipo de doença osteometabólica, faz-se necessário estabelecer o rastreamento dessa patologia. À vista disso, é cabível quantificar a massa óssea pela avaliação da densitometria mineral óssea, empregando-se a técnica de Absorciometria de Energia Dupla de Raios X ou DEXA (Dual Energy X-ray Absorptiometry). Conjectura-se que a redução da densidade mineral óssea tenha maior proeminência nos ossos longos e nas vértebras. Por tal razão, o exame DEXA é realizado no fêmur e na coluna lombar (L1-L4), justamente, sítios os quais estão mais susceptíveis à fratura. Além de serem regiões que são suficientes para refletir as condições de toda a estrutura esquelética (Dourado, 2012).

Com relação ao fêmur, a concentração de esforço na região da epífise proximal torna-se um fator de risco às fraturas nessa localidade, por tratar-se de uma estrutura na qual há, sobre ela, a descarga do peso corporal na posição ortostática, além de atuações de esforços dinâmicos, como exemplo, deambulação, corrida e outras atividades de impacto. Essa extremidade superior do fêmur apresenta acidentes anatômicos, tais como, cabeça, colo e ângulo de inclinação entre os eixos longitudinais do corpo e do colo, que são responsáveis por serem pontos concentradores de tensão (Moore et al., 2017). 
Destaca-se que o ângulo de inclinação tende a reduzir na idade avançada, fato que acentua ainda mais a concentração de esforços nessa área. Além disso, esse ângulo tende a ser menor nas mulheres, em virtude na pelve feminina ser mais larga e o corpo do fêmur ser mais obliquo (Moore et al., 2017). Portanto, esses achados anatômicos, associados à senectude, provavelmente, são responsáveis por maior incidência de fratura espontânea ou traumática nessa região, de modo especial, na população feminina pelos motivos explicitados.

A longevidade, sobremaneira, faz parte da realidade da sociedade contemporânea por diversas razões, como avanço da medicina, melhorias das condições sanitárias, planos de imunização, entre outros, que reúnem condições que promovem a qualidade de vida e, consequentemente, contribuem com o aumento do tempo médio de vida. Por outro lado, repercussões clínicas em virtude do envelhecimento urgem estudos para oferecer intervenções com substancial intuito de assegurar a manutenção do bem-estar e da saúde desse expoente grupo (Mota et al., 2012).

Em acréscimo, é importante citar que existem outros fatores fisiolopatológicos que podem desencadear a osteoporose, os quais não se restringem unicamente à população geriátrica. De acordo com Dourado (2012), mulheres atletas com carga de exercício físico extenuante, associado à maior massa magra e diminuta disponibilidade de tecido adiposo, podem sofrer privação dos ciclos menstruais, tornando-se susceptíveis a desenvolver osteoporose por alteração no padrão hormonal (Dourado, 2012). Ramalho e Castro (1999) descrevem, ainda, outras situações que também podem desencadear menopausa precoce, por exemplo, necessidade de ooforectomia bilateral e histerectomia total e anexos, as quais expõem mulheres jovens ao processo de supressão hormonal e, fatidicamente, à osteoporose (Ramalho \& Lazaretti-Castro, 1999).

As fraturas traumáticas de alta energia por acidentes de diversas naturezas também exigem atenção a outro grupo populacional. Por via de regra, esse grupo é composto majoritariamente por homens jovens economicamente ativos, os quais podem estar sujeitos à redução cirúrgica das fraturas diafisárias, transtrocantéricas e de colo do fêmur. Isoladamente, cada uma dessas fraturas apresentam desafio para o tratamento, principalmente, as de colo de fêmur, quanto à fixação e evolução clínica (Astur Neto et al., 2010).

Desse modo, independente, do perfil da pessoa, todos requerem, em semelhante proporção, qualidade de vida, reabilitação rápida, recuperação funcional, perspectiva de retorno às atividades profissionais, redução da ocorrência de anos potenciais de vida perdidos e ausência de sequelas ou as mínimas possíveis. Todos esses itens são quesitos essenciais, que devem ser priorizados na especificação dos dispositivos de fixação. A finalidade do rigor no desenvolvimento desses implantes reside na imprescindibilidade de que esses não apresentem falhas em uso e, consequentemente, evitar a necessidade de ressubmeter a pessoa a novo procedimento.

Diante dos fatos expostos, nota-se o quão relevante é a investigação das causas que levam à falha dos dispositivos de osteossíntese, com intuito de propor estudos para correção dos parâmetros identificados e otimização do projeto (desenho, dimensionamento, montagem e seleção de material), com objetivo de aperfeiçoar as técnicas de manufaturas e favorecer melhor prognóstico clínico pós-cirúrgico, minimizar recidivas de procedimentos cirúrgicos, diminuir o afastamento das atividades laborais, evitar sequelas permanentes e invalidez.

\section{Metodologia}

Realizou-se uma revisão integrativa da literatura (Estrela, 2018; Koche, 2011; Lüdke \& André, 1986; Pereira et al., 2018; Yin, 2014), cuja questão norteadora foi: quais motivos levam implantes de quadril a falharem em uso? A resposta à essa questão permitiu identificar os campos de estudos relacionados a implantes de quadril que demandam aprofundamento, e, portanto, novas possibilidades de pesquisas.

Os dados foram coletados através do Portal de Periódicos da CAPES, todas as bases da dados da CAPES foram 
utilizadas, dentre as quais, destacam-se: Scopus, Web of Science, PubMed, Springer, ScienceDirect. Cinco buscas foram realizadas nas bases de dados, utilizando como instrumentos de busca os descritores: "failure" e "hip implant"; "failure" e "hip implants"; "failure" e "hip replacement"; "failure" e "hip prosthesis"; e finalmente "failure" e "hip divaiced". Utilizando o operador booleano "AND". Os seguintes critérios de restrição foram inseridos: termos de busca presentes no título do artigo; artigos publicados na língua inglesa; artigos revisados por pares. Não foram impostas restrições quanto ao ano de publicação do artigo.

\section{Resultados e Discussão}

Após a associação de todos os descritores nas bases pesquisadas foram encontrados 88 artigos. Destes, 17 foram classificados como comunicações curtas, que abrangem resumos e cartas ao editor. Os 71 documentos restantes tratavam-se de artigos completos, após leitura identificou-se que 37 artigos tratavam-se de especificamente de falhas em implantes de quadril e os demais de temas associados. Os 37 artigos diretamente relacionados ao objeto de estudo deste artigo são apresentados nesta seção. Alguns dos outros 34 artigos relacionados ao tema também foram utilizados para complementar alguns temas abordados.

O artigo completo mais antigo localizado pela busca foi publicado por Martens e colaboradores em 1974 na revista Acta Orthopaedica (Martens et al., 1974). O artigo registra o grande sucesso e ritmo crescente da utilização do implante total de quadril na época, contudo, os autores também ressaltam que apesar do sucesso da técnica, o desempenho dos implantes à longo prazo ainda era pouco conhecido. Os autores apontaram que, na época, estudos sobre problemas de desgaste das superfícies articuladas e questões de fixação eram realizados, contudo pouco se conhecia sobre o processo de fadiga nos implantes. Nessa linha, o artigo apresentou seis implantes que falharam por fadiga. Fraturas por fadiga são relatadas ao longo dos anos, incluindo o artigo mais recente localizado pela revisão, publicado este ano por Pimenta e colaboradores. (Pimenta et al., 2021).

\subsection{O implante}

Apesar de decorridas décadas do início da utilização dos implantes, e dos intensos esforços para o desenvolvimento e aperfeiçoamento dos materiais, ainda não se chegou a um material, que pudesse receber o título de padrão ouro para utilização em implantes. Todas as propostas realizadas até o momento, em maior ou menor quantidade apresentaram falhas. Estudos relatam casos de falhas em implantes fabricados na liga Protasul liga (Co-Cr-Mo) (Martens et al., 1974; Pazzaglia et al., 1988); vitallium (liga Co-Cr-Mo) (Pizzoferrato et al., 1988); aço inoxidável 316L (Godec, 2011; Jakobsen et al., 2014; Kiani Khouzani et al., 2018; Maheshwari et al., 2012; Pazzaglia et al., 1988); aço inoxidável ASTM F-745 (Griza et al., 2008); aço inoxidável F-183 (Hernandez-Rodriguez et al., 2010); liga de titânio (Ceretti \& Falez, 2016; Grupp et al., 2010; Jakobsen et al., 2014; Oshima \& Fetto, 2015; Paydar et al., 2007; Pizzoferrato et al., 1988); liga Ti-6Al-4V (Chao \& López, 2007; Cicero et al., 2007; Jakobsen et al., 2014; Moretti et al., 2012; Pimenta et al., 2021; Williams \& Chawla, 2014); fibra de carbono (Adam et al., 2002).

\section{Fadiga}

Estudos realizados em 1974 e 1988 indicam a baixa resistência à fadiga da liga Co-Cr, denominada Protasul, (Martens et al., 1974; Pazzaglia et al., 1988). Em 1974 Martens e colaboradores, relataram que ao utilizar um lote de implantes fabricados com a liga Protasil, seis próteses de um total de 56 implantadas falharam por fadiga. Já quando um lote anterior de implantes fabricados em aço inoxidável havia sido utilizado, não tinham ocorrido falhas dessa natureza. O melhor desempenho 
do aço inoxidável foi atribuído ao seu maior limite de escoamento, $680 \mathrm{MPa}$, contra $300 \mathrm{MPa}$ da liga à base de Co-Cr (Martens et al., 1974). Embora o limite de escoamento não seja o único fator a influenciar no desempenho à fadiga de um material, certamente desempenha um papel primordial no aumento da resistência à fadiga (ASM, 1990, 1996). Entretanto, implantes de aço inoxidável também estão sujeitos a falhas por fadiga, artigo de Hernandez-Rodriguez e colaboradores atribuíram à existência de uma descontinuidade superficial uma falha por fadiga ocorrida em um implante de aço inoxidável (HernandezRodriguez et al., 2010).

Diversos outros estudos apontam fraturas por fadiga nas hastes dos implantes, em geral algum defeito é responsável por iniciar o processo de fadiga. Cicero e colaboradores identificaram que um implante fabricado na liga Ti-6Al-4V fraturou por fadiga devido a inclusões de alumina presentes na interface do implante com o revestimento poroso de titânio puro. A aplicação desses revestimentos foi utilizada para promover a osteointegração (Cicero et al., 2007). Embora os autores não tenham indicado, a presença de inclusões de alumina é comum em peças com revestimento. Uma etapa de preparação para o revestimento consiste no jateamento do componente com partículas de alumina, fato que resulta na incrustação de algumas dessas partículas (Campos et al., 2020). Pimenta e colaboradores identificaram que um processo de corrosão deu origem a pequenos pits na superfície do implante, os quais foram responsáveis por iniciar o processo de fadiga. Segundo os autores, a corrosão ocorreu porque o implante que era fabricado na liga Ti-6Al-4V possuía em sua microestrutura uma fase que prejudica a resistência à corrosão (Pimenta et al., 2021). Fenômeno similar ao observado por Pimenta e colaboradores (Pimenta et al., 2021) também foi observado por Lanzutti e colaboradores em um implante fabricado com uma liga Co-Cr-Mo (Lanzutti et al., 2019). Kiani Khouzani e colaboradores identificam que um implante fabricado em aço inoxidável AISI 316L falhou devido a um erro metalúrgico na fabricação do implante, que resultou em um excesso de inclusões. As inclusões atuam como concentradores de tensão, o que resultou na falha por fadiga do implante (Kiani Khouzani et al., 2018).

Williams e Chawla estudaram um caso de falha ocorrida em um implante bi-modular fabricado na liga Ti-6Al-4V. Os autores concluíram que durante a cirurgia de colocação do implante não foi utilizada a força necessária para ajustar a junta cônica, o que resultou na formação de pits pela associação de corrosão por frestas e desgaste por atrito. Fato que reduziu drasticamente a vida à fadiga do implante (Williams \& Chawla, 2014). A formação de pits de corrosão por frestas também foi ratificada em estudo realizado por Ceretti e Falez (Ceretti \& Falez, 2016). A baixa resistência à fadiga de implantes modulares foi corroborada por Grupp e colaboradores (Grupp et al., 2010).

Estudos de Chao e López e de Griza e colaboradores identificaram que a geometria do implante pode ser decisiva na propagação de trincas por fadiga. No primeiro estudo, os autores apontaram que a geometria de transição da haste para a pescoço, resultava em um grande concentrador de tensão, fato que gerou a fratura por fadiga (Chao \& López, 2007). No segundo estudo, a utilização de sulcos transversais na haste, que originalmente deveriam impedir o afrouxamento da mesma, resultaram em um concentrador de tensão, propagando a trinca de fadiga (Griza et al., 2008). Grupp e colaboradores analisaram a falha de adaptadores modulares de pescoço para hastes de artroplastia. Esses adaptadores permitem que o cirurgião modifique o ângulo do implante, contudo os autores concluíram que os implantes fraturaram por fadiga, independentemente se fabricados com ligas de titânio ou Co-Cr (Grupp et al., 2010).

\section{Afrouxamento}

O afrouxamento da fixação do implante é uma falha comum de ocorrer, Ebramzadeh e colaboradores analisaram 433 implantes que apresentaram algum tipo de falha, o afrouxamento foi responsável por 172 desses casos, sendo o motivo mais frequente de falha (Ebramzadeh et al., 2011). Outros estudos analisaram o percentual da falha de afrouxamento em relação ao total de pacientes submetidos a artroplastia total de quadril: Norton e colaboradores relataram essa falha em 15 de um total de 29 cirurgias realizadas (Norton et al., 2002); Streit e colaboradores indicaram uma ocorrência de apenas 7\% de falhas 
associadas ao afrouxamento ao acompanharem 326 pacientes(Streit et al., 2011); Burke e colaboradores indicaram que o afrouxamento foi responsável por 42,3\% de um total de 1.062 cirurgias de revisão (Burke et al., 2019); o afrouxamento também foi apontado como o principal responsável pelas cirurgias de revisão em estudo publicado sobre 1.127 casos de cirurgia de revisão (Affatato et al., 2019). Contudo, nenhum dos estudos anteriores relacionou o ocorrido com o material utilizado na fabricação do implante.

Estudo realizado por Sott e Rosson apontou que os implantes fabricados em ligas de titânio possuem maior probabilidade de afrouxamento em um menor tempo (entre 5 e 16 anos - mediana 7,8), em relação as ligas de Co-Cr (entre 7 e 22 anos - mediana 13) (Sott \& Rosson, 2002). Em outro trabalho, Adam e colaboradores concluíram que próteses feitas com hastes de fibras de carbono apresentam ainda maior problema de afrouxamento. Os autores documentaram a necessidade de cirurgia de revisão em 47 implantes de um total de 51 realizados. O tempo de afrouxamento também foi menor que o mencionado para ligas de titânio e Co-Cr pelo estudo abordado anteriormente, entre 0,75 e 4,8 anos, com média de 2,5 (Adam et al., 2002). Godec relatou um caso de afrouxamento em um implante fabricado de aço inoxidável (Godec, 2011), sendo o fato atribuído a um erro metalúrgico na fabricação do material, que originou uma série de inclusões não metálicas.

\section{Metalose}

Outro grave problema que pode ocorrer em implantes é a metalose, termo clínico usado para definir a infiltração de resíduos metálicos provocando inflamações crônicas (Chang et al., 2005). Em sistemas onde existem movimento relativo entre as partes, como na artroplastia total de quadril, o atrito pode acarretar em desgaste dos componentes metálicos, provocando a liberação de partículas. Langton e colaboradores acompanharam um grupo de 660 pacientes submetidos a cirurgia de quadril com colocação de implantes, desses, 17 desenvolveram reações adversas devido à liberação de partículas metálicas (Langton et al., 2010). Paydar e colaboradores relataram um caso de metalose que resultou em uma cirurgia de revisão após 15 anos da colocação de um implante de liga de titânio (Paydar et al., 2007). Outra falha por metalose foi relatada por Toms e colaboradores (Toms et al., 2009).

Bolland e colaboradores associaram uma alta taxa de ocorrência de metalose em implantes metal-metal com grandes diâmetros. Os autores identificaram uma taxa de falha por metalose de 15,1\% das cirurgias realizadas em cinco anos (Bolland et al., 2011). Moretti e colaboradores, relataram um caso raro de neuropatia periférica grave causada pela absorção excessiva de vanádio proveniente da liga Ti-6Al-4V (Moretti et al., 2012).

Jakobsen e colaboradores observaram a influência da composição química da liga na metalose. Os autores constaram que o cromo é o elemento menos propenso à liberação de partículas, não tendo sido identificado nenhum caso. Para implantes à base de Cobalto, a metalose foi identificada em quatro de um total de 62 análises realizadas. E em implantes à base de níquel, um caso foi identificado em 24 dispositivos analisados (Jakobsen et al., 2014). Contudo, Thomas e Prieto identificaram a metalose de cobalto e cromo como a causa responsável pela falha de uma prótese (Thomas \& Prieto, 2019), demostrando que o cromo também deve ser um elemento a ser considerado nas análises. A liberação de partículas por ligas de cobalto também foi estudada por Hart e colaboradores (Hart et al., 2011), que propuseram um método para prever a falha por este mecanismo.

\section{Outras Causas}

A fratura também pode ocorrer no componente cerâmico. Faraj relatou dois casos de fraturas em componentes cerâmicos, incluindo um caso de fratura em múltiplos fragmentos devido a uma queda sofrida pelo paciente (Faraj, 2009). Ao analisar um caso de fraturas múltiplas em um componente de cerâmica, Moretti e colaboradores (Moretti et al., 2012) associaram a falha ao mal posicionamento do implante durante a cirurgia. 
A qualidade e a espessura do polietileno utilizado também são fundamentais para o sucesso da cirurgia, conforme relato de caso feito por Oshima e Fetto, que apontaram o polietileno como causa da falha de um implante metal-metal (Oshima \& Fetto, 2015). Em outro estudo Shahemi e colaboradores analisaram um implante que foi retirado após 22 anos de uso devido ao paciente queixar-se de dores, e constaram que o problema ocorreu devido ao desgaste do polietileno (Shahemi et al., 2018). A falha no polietileno também foi apontada por Thomas e colaboradores (Thomas et al., 2020) em outro estudo de caso, sendo um problema digno de mais estudos.

Embora o aço inoxidável possua propriedades de resistência mecânica adequada, a resistência à corrosão pode não ser a necessária para a aplicação em implantes. Pazzagliae colaboradores, analisaram 9 implantes fabricados com aço inoxidável 316 que falharam em uso, os quais representavam 2,4\% do total de 365 intervenções realizadas. Os autores constataram que a origem de todas as falhas foi a existência de um processos corrosivo na liga (Pazzaglia et al., 1988).

\subsection{Pacientes}

Estudo realizado com a população dinamarquesa entre os anos de 1995 e 2002 com 36.984 pacientes (21.707 mulheres e 15.277 homens) submetidos à cirurgia, demostrou que o sexo masculino é um fator fortemente preditivo de falhas em implantes de quadril. As mulheres apresentaram um índice de 2,8\% de demanda por revisões, enquanto nos homens esse índice foi de 3,4\% (Johnsen et al., 2006). Outro estudo publicado por Ebramzadeh e colaboradores apontaram para a ausência de uma relação entre o sexo e o índice de falhas. Neste estudo foram analisadas 433 situações de falhas, das quais os homens respondiam por 189 e as mulheres por 201 (Ebramzadeh et al., 2011). Contudo, chama-se atenção para o fato de os estudos terem utilizado metodologias diferentes, enquanto o primeiro verificou o número de falhas por sexo sobre o total de cirurgias realizadas (Johnsen et al., 2006), o segundo verificou o percentual de falhas em cada sexo, considerando apenas os casos de falhas (Ebramzadeh et al., 2011).

Quando, contudo, analisa-se, especificamente, a falha por metalose, as mulheres apresentam maior prevalência (Langton et al., 2010), essa hipótese é corroborada por um amplo estudo realizado por Ebramzadeh e colaboradores, que ao analisarem 433 casos de falhas (189 de homens e 203 de mulheres), identificaram que as mulheres tiveram 6,6 vezes mais casos de metalose que os homens, 20 versus 3 casos, respectivamente.

Streit e colaboradores acompanharam um total de 326 pacientes submetidos a artroplastia total de quadril durante um período médio de 17 anos após a cirurgia. Durante os estudos ocorreram cirurgias de revisão em 86 casos. Os autores não encontraram uma relação entre as fraturas e a idade dos pacientes (Streit et al., 2011). Entretanto, a existência de uma relação é controversa, estudo realizado por Johnsen e colaboradores identificou uma relação entre a idade e o índice de falhas de implantes de quadril, sendo atribuído maior risco nos primeiros meses à maior idade, enquanto falhas em períodos posteriores estariam associadas à pacientes mais jovens. Nos primeiros 30 dias após a operação, uma idade acima de 80 anos foi associada a um aumento no risco de falha. Contudo, ter menos de 60 anos foi associado a um maior risco de falhas no período entre seis meses e 8,6 anos após a cirurgia (Johnsen et al., 2006).

A idade também é associada como fator de risco para falhas por fadiga, pacientes mais jovens, e, por consequência, mais ativos fisicamente estão mais sujeitos às falhas por fadiga (Hernandez-Rodriguez et al., 2010). A fadiga por ser definida como um processo de mudança estrutural localizada e progressiva que ocorre devido à aplicações de tensões que flutuam de intensidade com o tempo, resultando na formação e crescimento de trincas, podendo acarretar à fratura do material após um determinado número de flutuações (ASM, 1996). Desta forma, observa-se que a fratura por fadiga, apenas ocorrerá após um certo número de flutuações, estando esse mecanismo associado a fraturas tardias, em geral, após alguns anos. Corroborando, assim, estudo de Johnsen apresentado anteriormente, que relacionou uma predominância de falhas em períodos posteriores à pacientes mais jovens (Johnsen et al., 2006). 
Estudo de Jamsen e colaboradores analisaram a influência do sistema de fixação das próteses em pessoas de idades avançadas. Os autores constaram que próteses não cimentadas resultavam em um risco maior de cirurgias de revisão nos primeiros anos. Contudo, para períodos mais longos e índices de mortalidade, o sistema de fixação não demostrou influência (Jämsen et al., 2014).

\section{Considerações Finais}

Sob o prisma das questão apresentadas, ao analisar o leque dessas repercussões clínicas, a fratura óssea, indubitavelmente, reside em um dos principais problemas associados ao aumento da expectativa de vida da população (Caputo \& Costa, 2014). Principalmente, as fraturas femorais, as quais demandam por tratamento cirúrgico para redução da fratura por osteossíntese. Trata-se de uma intervenção custosa, pois além dos gastos intrínsecos à cirurgia e à internação, soma-se também o custo atribuído à aquisição dos dispositivos de fixação. Em acréscimo, consiste em uma intervenção a qual requer processo de recuperação mais prolongado, o tempo de hospitalização do paciente pode ser mais longo, assim como, maior período de ocupação de leito. Além da exposição à riscos de infecção hospitalar e demais complicações inerentes ao próprio procedimento cirúrgico, de modo geral.

É relevante, portanto, o fato de que os dispositivos de osteossíntese precisam ser elaborados de forma multidisciplinar com parâmetros exigentes, envolvendo ciência médica, projeto de engenharia e seleção de material, com propósito de minorar os riscos de falhas e prevenir acidentes, por conseguinte, evitar ressubmeter o indivíduo a novo procedimento cirúrgico de correção. Dependendo das infecções que tenham ocorrido com a dispositivo fraturado, pode ser necessário um procedimento em duas etapas, uma cirurgia para retirada do dispositivo danificado e uma segunda para implantação de um novo, com antibioticoterapia no intervalo (Pizzoferrato et al., 1988). Ressalta-se que a falha de uma prótese implica, necessariamente, na re-hospitalização do paciente, fato que sobressai a preocupação relativa ao prognóstico da evolução clínica. Tendo em vista que fraturas de quadril, em geral, são as que mais contribuem com a morbidade e a mortalidade (Caputo \& Costa, 2014).

Nessa perspectiva, no que tange a taxa de sobrevida, é sabido que a morbidade operatória restringe o paciente ao leito, no caso do idoso, quando acamado, ele fica mais susceptível à depressão, quadro clínico que dificulta a recuperação, que, infortunadamente, pode contribuir com desfechos não favoráveis, como desinteresse na reabilitação, prejuízo na qualidade de vida, debilidade e óbito. Sendo necessários estudos que aumentem a eficácia e confiabilidade dos implantes de quadril.

A revisão aqui apresentada indicou a necessidade de avanço em estudos para o desenvolvimento de novos materiais, menos suscetíveis à corrosão quando implantados no corpo humano. Os metais atualmente utilizados, ligas de Ti, ligas de Co e aços inoxidáveis apresentam problemas de corrosão que podem resultar em metalose e falhas por fadiga. A tentativa da utilização de fibra de carbono não foi bem sucedida. Estudos de corrosão in vitro são um caminho para o aprimoramento desses materiais (Runa et al., 2017)

Outro ponto importante a ser estudado, é o processo de fadiga, que é responsável por diversas falhas em implantes de quadril. O desenvolvimento de ligas com maior resistência à fadiga é necessário, assim como, cuidados no acabamento superficial dos implantes, que podem vir a resultar em concentradores de tensão, diminuindo drasticamente a vida do componente. A geometria dos implantes também desempenha papel fundamental na resistência à fadiga. Nessa linha, a condução de estudos que otimizem a geometria dos implantes de acordo com as especificidades anatômicas do paciente, tornase parâmetro primordial na otimização de projeto dos implantes. Estudos utilizando análise por elementos finitos podem ser apontados como um caminho para pesquisas de geometria e falhas por fadiga (Babić et al., 2020; Bingol et al., 2011; Ferrand, 2018; Steinhauser et al., 2006).

Em síntese, ao reunir as técnicas de aprimoramento de projeto de engenharia, é importante que os cirurgiões- 
ortopedistas, que fazem parte dessa equipe multidisciplinar, interliguem otimização de projeto e variação anatômica, a fim de selecionar o modelo de dispositivo mais adequado para cada paciente, considerando características importantes como a faixa etária, expectativa de vida, biotipo e perfil de atividades físicas.

\section{Referências}

Adam, F., Hammer, D. S., Pfautsch, S., \& Westermann, K. (2002). Early failure of a press-fit carbon fiber hip prosthesis with a smooth surface. Journal of Arthroplasty, 17(2), 217-223. https://doi.org/10.1054/arth.2002.30285

Affatato, S., Cosentino, M., Castagnini, F., \& Bordini, B. (2019). Registry study on failure incidence in 1,127 revised hip implants with stem trunnion re-use after 10 years of follow-up: limited influence of an adapter sleeve. Acta Orthopaedica, 90(5), 417-420. https://doi.org/10.1080/17453674.2019.1618649

ASM. (1990). ASM handbook, Vol. 1 properties and selection: irons, steels, and high-performance alloys. ASM International.

ASM. (1996). ASM handbook vol19 Fatigue and fracture. ASM International.

Astur Neto, N., Lins, R. A. B., Kojima, K. E., Cunha, B. L. da, Hungria Neto, J. S., Mercadante, M. T., Christian, R. W., \& Hungria, J. O. S. (2010). Resultados do tratamento das fraturas da diáfise do fêmur ipsilaterais às do colo ou transtrocantérica. Acta Ortopédica Brasileira, 18(5), 255-260. https://doi.org/10.1590/S1413-78522010000500004

Babić, M., Verić, O., Božić, Ž., \& Sušić, A. (2020). Finite element modelling and fatigue life assessment of a cemented total hip prosthesis based on 3D scanning. Engineering Failure Analysis, 113(November 2019). https://doi.org/10.1016/j.engfailanal.2020.104536

Bingol, M., Kaymaz, I., \& Karsan, O. (2011). The effects of the cement thickness on the failure probability of the cemented hip prosthesis. Journal of Biomechanics, 44, 9. https://doi.org/10.1016/j.jbiomech.2011.02.041

Bolland, B. J. R. F., Culliford, D. J., Langton, D. J., Millington, J. P. S., Arden, N. K., \& Latham, J. M. (2011). High failure rates with a large-diameter hybrid metal-on-metal total hip replacement: Clinical, radiological and retrieval analysis. Journal of Bone and Joint Surgery - Series B, 93 B(5), 608-615. https://doi.org/10.1302/0301-620X.93B5.26309

Burke, N. G., Gibbons, J. P., Cassar-Gheiti, A. J., Walsh, F. M., \& Cashman, J. P. (2019). Total hip replacement- the cause of failure in patients under 50 years old? Irish Journal of Medical Science, 188(3), 879-883. https://doi.org/10.1007/s11845-018-01956-8

Campos, P. K., Cardoso, B. R., Furtado, H. C., Pimenta, A. R., \& Diniz, M. G. (2020). Characterization of mechanical and surface finishing properties of metallic coating obtained by arc electric thermal spray. Brazilian Journal of Development, 6(3), 11078-11090. https://doi.org/10.34117/bjdv6n3-106

Caputo, E. L., \& Costa, M. Z. (2014). Influência do exercício físico na qualidade de vida de mulheres pós-menopáusicas com osteoporose. Revista Brasileira de Reumatologia, 54(6), 467-473. https://doi.org/10.1016/j.rbr.2014.02.008

Ceretti, M., \& Falez, F. (2016). Modular titanium alloy neck failure in total hip replacement: Analysis of a relapse case. Sicot-J, 2(Figure 3), 3-6. https://doi.org/10.1051/sicotj/2016009

Chang, J.-D., Lee, S.-S., Hur, M., Seo, E.-M., Chung, Y.-K., \& Lee, C.-J. (2005). Revision Total Hip Arthroplasty in Hip Joints With Metallosis: a singlecenter experience with 31 cases. The Journal of Arthroplasty, 20(5), 568-573. https://doi.org/10.1016/j.arth.2005.04.001

Chao, J., \& López, V. (2007). Failure analysis of a Ti6Al4V cementless HIP prosthesis. Engineering Failure Analysis, 14(5), 822-830. https://doi.org/10.1016/j.engfailanal.2006.11.003

Cicero, S., Gutiérrez-Solana, F., Álvarez, J. A., \& Sánchez, L. (2007). Failure analysis of a hip implant by using the FITNET fitness for service procedure. Engineering Fracture Mechanics, 74(5), 688-702. https://doi.org/10.1016/j.engfracmech.2006.06.020

Dourado, C. M. (2012). Densidade mineral óssea em idosos e presença de fatores de risco nutricionais para osteoporose. Pontifícia Universidade Católica do Rio Grande do Sul.

Ebramzadeh, E., Campbell, P. A., Takamura, K. M., Lu, Z., Sangiorgio, S. N., Kalma, J. J., De Smet, K. A., \& Amstutz, H. C. (2011). Failure Modes of 433 Metal-on-Metal Hip Implants: How, Why, and Wear. Orthopedic Clinics of North America, 42(2), 241-250. https://doi.org/10.1016/j.ocl.2011.01.001

Estrela, C. (2018). Metodologia científica: ciência, ensino, pesquisa $\left(3^{\circ}\right)$. Artes Médicas.

Faraj, A. (2009). Patterns of failure of ceramic liner in total hip replacement: Report of two cases. European Journal of Orthopaedic Surgery and Traumatology, 19(1), 43-46. https://doi.org/10.1007/s00590-008-0365-y

Ferrand, H. L. (2018). Multiscale modeling speedily predicts fatigue in hip implants. MRS Bulletin, 43(July), 472.

Gatner, L. P. (2007). Tratado de Histologia em cores . Elsevier.

Godec, M. (2011). Material failure of an AISI 3161 stainless steel hip prosthesis. Materiali in Tehnologije, 45(2), 85-90.

Griza, S., Kwietniewski, C., Tarnowski, G. A., Bertoni, F., Reboh, Y., Strohaecker, T. R., \& Baumvol, I. J. R. (2008). Fatigue failure analysis of a specific total hip prosthesis stem design. International Journal of Fatigue, 30(8), 1325-1332. https://doi.org/10.1016/j.ijfatigue.2007.11.005

Grupp, T. M. M. titanium alloy neck adapter failures in hip replacement-F. mode analysis and influence of implant material, Weik, T., Bloemer, W., \& 
Knaebel, H. P. (2010). Modular titanium alloy neck adapter failures in hip replacement - Failure mode analysis and influence of implant material. BMC Musculoskeletal Disorders, 11, 1-13. https://doi.org/10.1186/1471-2474-11-3

Hart, A. J., Sabah, S. A., Bandi, A. S., Maggiore, P., Tarassoli, P., Sampson, B., \& Skinner, J. A. (2011). Sensitivity and specificity of blood cobalt and chromium metal ions for predicting failure of metal-on-metal hip replacement. Journal of Bone and Joint Surgery - Series B, 93 B(10), 1308-1313. https://doi.org/10.1302/0301-620X.93B10.26249

Hernandez-Rodriguez, M. A. L., Ortega-Saenz, J. A., \& Contreras-Hernandez, G. R. (2010). Failure analysis of a total hip prosthesis implanted in active patient. Journal of the Mechanical Behavior of Biomedical Materials, 3(8), 619-622. https://doi.org/10.1016/j.jmbbm.2010.06.004

Jakobsen, S. S., Lidén, C., Søballe, K., Johansen, J. D., Menné, T., Lundgren, L., Bregnbak, D., Møller, P., Jellesen, M. S., \& Thyssen, J. P. (2014). Failure of total hip implants: Metals and metal release in 52 cases. Contact Dermatitis, 71(6), 319-325. https://doi.org/10.1111/cod.12275

Jämsen, E., Eskelinen, A., Peltola, M., \& Mäkelä, K. (2014). High early failure rate after cementless hip replacement in the octogenarian. Clinical Orthopaedics and Related Research, 472(9), 2779-2789. https://doi.org/10.1007/s11999-014-3641-7

Johnsen, S. P., Sørensen, H. T., Pedersen, A. B., Lucht, U., Søballe, K., \& Overgaard, S. (2006). Patient-related predictors of implant failure after primary total hip replacement in the initial, short- and long-term: A nationwide Danish folow-up study including 36984 patients. Journal of Bone and Joint Surgery - Series B, 88(10), 1303-1308. https://doi.org/10.1302/0301-620X.88B10.17399

Kiani Khouzani, M., Bahrami, A., \& Eslami, A. (2018). Metallurgical aspects of failure in a broken femoral HIP prosthesis. Engineering Failure Analysis, 90(November 2017), 168-178. https://doi.org/10.1016/j.engfailanal.2018.03.018

Koche, J. C. (2011). Fundamentos de metodologia científica. Editora Vozes. https://doi.org/10.1590/S1517-97022003000100005

Langton, D. J., Jameson, S. S., Joyce, T. J., Hallab, N. J., Natu, S., \& Nargol, A. V. F. (2010). Early failure of metal-on-metal bearings in hip resurfacing and large-diameter total hip replacement: A consequence of excess wear. Journal of Bone and Joint Surgery - Series B, 92(1), 38-46. https://doi.org/10.1302/0301620X.92B1.22770

Lanzutti, A., Andreatta, F., Rossi, L., Di Benedetto, P., Causero, A., Magnan, M., \& Fedrizzi, L. (2019). Corrosion fatigue failure of a high carbon CoCrMo modular hip prosthesis: Failure analysis and electrochemical study. Engineering Failure Analysis, 105(July), 856-868. https://doi.org/10.1016/j.engfailanal.2019.07.044

Lüdke, M., \& André, M. E. D. A. (1986). Pesquisa em educação: abordagens qualitativas. UPU. https://edisciplinas.usp.br/pluginfile.php/4091392/mod_resource/content/1/Lud_And_cap3.pdf.

Maheshwari, A. V., Chawla, A., Osuji, O. U., Malhotra, R., \& Gulati, Y. P. (2012). Fracture of the outer metallic head of the bipolar hip prosthesis: An unusual bearing surface failure. Journal of Arthroplasty, 27(2), 323.e9-323.e12. https://doi.org/10.1016/j.arth.2011.03.018

Martens, M., Aernoudt, E., Meester, P. De, Ducheyne, P., Mulier, J. C., Langh, R. De, \& Kestelijn, P. (1974). Factors in the mechanical failure of the femoral component in total hip prosthesis: Report of six fatigue fractures of the femoral stern and results of experimental loading tests. Acta Orthopaedica, 45(5), 693710. https://doi.org/10.3109/17453677408989679

Moore, K. L., Dalley, A. F., \& Agur, A. M. R. (2017). Anatomia voltada para clínica. Guanabara Koogan.

Moretti, B., Pesce, V., MacCagnano, G., Vicenti, G., Lovreglio, P., Soleo, L., \& Apostoli, P. (2012). Peripheral neuropathy after hip replacement failure: Is vanadium the culprit? The Lancet, 379(9826), 1676. https://doi.org/10.1016/S0140-6736(12)60273-6

Mota, L. S., Sousa, E. G., \& Azevedo, F. H. C. (2012). Intercorrências da osteoporose na qualidade de vida dos idosos. Revista Interdisciplinar NOVAFAPI, $5(2), 44-49$.

Norton, M. R., Yarlagadda, R., \& Anderson, G. H. (2002). Catastrophic failure of the Elite Plus total hip replacement, with a Hylamer acetabulum and Zirconia ceramic femoral head. Journal of Bone and Joint Surgery - Series B, 84(5), 631-635. https://doi.org/10.1302/0301-620X.84B5.12679

Oshima, Y., \& Fetto, J. F. (2015). Mechanical failure of metal-polyethylene sandwich liner in metal-on-metal total hip replacement. Journal of Materials Science: Materials in Medicine, 26(1). https://doi.org/10.1007/s10856-015-5383-9

Paydar, A., Chew, F. S., \& Manner, P. A. (2007). Severe Periprosthetic Metallosis and Polyethylene Liner Failure Complicating Total Hip Replacement: The Cloud Sign. Radiology Case Reports, 2(4), 115. https://doi.org/10.2484/rcr.v2i4.115

Pazzaglia, U. E., Ghisellini, F., Barbieri, D., \& Ceciliani, L. (1988). Failure of the stem in total hip replacement - A study of aetiology and mechanism of failure in 13 cases. Archives of Orthopaedic and Traumatic Surgery, 107(4), 195-202. https://doi.org/10.1007/BF00449667

Pereira, A. S., Shitsuka, D. M. S., Parreira, F. J., \& Shitsuka, R. (2018). Metodologia da pesquisa científica. UAB/NTE/UFSM. https://repositorio.ufsm.br/bitstream/handle/1/15824/Lic_Computacao_Metodologia-Pesquisa-Cientifica.pdf?sequence=1. Acesso em: 28 março 2020.

Pimenta, A. R., Tavares, S. S. M., Dias, D. F., Correa, S. R., Sobreiro, A. L., \& Diniz, M. G. (2021). Failure analysis of a titanium hip prosthesis. Journal of Failure Analysis and Prevention, 21(1), 28-35. https://doi.org/10.1007/s11668-020-01041-2

Pizzoferrato, A., Savarino, L., Stea, S., \& Tarabusi, C. (1988). Results of histological grading on 100 cases of hip prosthesis failure. Biomaterials, 9(4), 314318. https://doi.org/10.1016/0142-9612(88)90025-7

Ramalho, A. C., \& Lazaretti-Castro, M. (1999). Fisiopatologia da Osteoporose Involutiva. Arq Bras Endocrinol Metab, 43(6), $409-414$.

Runa, M., (Jenny) Lau, E. L., Takoudis, C., Sukotjo, C., Shokuhfar, T., Rocha, L., \& Mathew, M. (2017). In vitro Evaluation of Tribocorrosion Induced Failure Mechanisms at the Cell-Metal Interface for the Hip Implant Application. Advanced Engineering Materials, 19(5), 1-16. 
Research, Society and Development, v. 10, n. 11, e357101119668, 2021

(CC BY 4.0) | ISSN 2525-3409 | DOI: htttp://dx.doi.org/10.33448/rsd-v10i11.19668

https://doi.org/10.1002/adem.201600797

Shahemi, N., Liza, S., Abbas, A. A., \& Merican, A. M. (2018). Long-term wear failure analysis of uhmwpe acetabular cup in total hip replacement. Journal of the Mechanical Behavior of Biomedical Materials, 87(July), 1-9. https://doi.org/10.1016/j.jmbbm.2018.07.017

Sott, A. H., \& Rosson, J. W. (2002). The influence of biomaterial on patterns of failure after cemented total hip replacement. International Orthopaedics, 26(5), 287-290. https://doi.org/10.1007/s00264-002-0367-z

Steinhauser, E., Eichhorn, S., Schreiber, U., \& Gradinger, R. (2006). Failure analysis of revision implants for total hip replacement. Journal of Biomechanics, 39 (Suppl 1), S512.

Streit, M. R., Merle, C., Clarius, M., \& Aldinger, P. R. (2011). Late peri-prosthetic femoral fracture as a major mode of failure in uncemented primary hip replacement. Journal of Bone and Joint Surgery - Series B, 93 B(2), 178-183. https://doi.org/10.1302/0301-620X.93B2.24329

Thomas, W. C., Parvataneni, H. K., Vlasak, R. G., \& Gray, C. F. (2020). Early Polyethylene Failure in a Modern Total Hip Prosthesis: A Note of Caution. Journal of Arthroplasty, 35(5), 1297-1302. https://doi.org/10.1016/j.arth.2019.12.043

Thomas, W. C., \& Prieto, H. A. (2019). Total hip replacement failure due to adverse local tissue reaction from both ceramic abrasive wear and trunnion corrosion. Arthroplasty Today, 5(4), 384-388. https://doi.org/10.1016/j.artd.2019.10.001

Toms, A. P., Nolan, J., Barker, T., Darrah, C., \& Malcolm, P. (2009). Early failure of a Birmingham resurfacing hip replacement with lymphoreticular spread of metal debris: pre-operative diagnosis with MR. The British Journal of Radiology, 82(977), e87-e91. https://doi.org/10.1259/bjr/32928628

Williams, J. J., \& Chawla, N. (2014). Fractography of a neck failure in a double-modular hip implant. Case Studies in Engineering Failure Analysis, 2(1), 4550. https://doi.org/10.1016/j.csefa.2014.03.001

Yin, R. K. (2014). Estudo de caso: planejamento e métodos $\left(5^{\circ}\right)$. Bookman. 